\title{
Coordinate Transformation Technology in the Non-Normal Shutdown Positioning System
}

\author{
Hongwei Zhang, a and Jiawei Liu, b \\ ${ }^{1}$ Southwest University for Nationalities, Electrical and Information Engineering, Chengdu, China \\ a759724337@qq.com, b979464265@qq.com
}

Keywords: Highway; Coordinate transformation; Assembly line; Position monitoring

\begin{abstract}
The main research and coordinate the radar positioning system based on the translation of the highway algorithm for common coordinate system for vehicle position coordinate data of the non-normal stop on the highway coordinate transformation, in order to complete the vehicle monitoring, the algorithm Cordic architecture thinking, complex iterative mathematical relationship to the results of the hardware can be implemented to improve the efficiency of the system, and to adopt a pipeline structure in order to increase the speed of the algorithm to meet the requirements of real-time control of the system, the related software the structure of the algorithm and simulation, and FPGA platform to achieve coordinate transformation algorithm, error results are analyzed to meet system requirements.
\end{abstract}

\section{坐标转换技术在非正常停车定位系统中的应用}

\author{
张红伟 ${ }^{1 . a}$, 刘家玮 ${ }^{1 . b}$ \\ 1. 西南民族大学电气信息工程学院, 中国 成都市 610225
}

a759724337@qq.com, b979464265@qq.com

摘要: 主要研究了针对高速公路并基于雷达定位系统的坐标转换算法, 针对常用的坐标系统, 对高速公路上非正常停车的车辆位置坐标数据进行坐标转换, 以此来完成对车辆监控, 该算 法基于 Cordic 架构思想, 将复杂的数学关系迭代成硬件可实现的结果, 提高了系统的效率, 并对该算法采取一种流水线的结构以此来增加速度, 满足该系统的实时控制的要求, 在相关 软件上对算法和结构进行了仿真, 并在 FPGA 平台实现了坐标转换的算法, 对误差结果进行了 分析, 满足系统要求。

关键词: 高速公路; 坐标转换 ; 流水线; 位置监控

\section{1. 引言}

在高速公路上经常有机动车无故停车或者占用应急道, 并且在大雾雨雪天气, 视线模糊的情 况下，未免事故的发生，需对高速公路车辆定位并报警，在对高速公路上的车辆位置进行实 时监控时, 需要进行对车辆进行定位, 将定位所得到的坐标送入终端系统, 进行坐标转换。 在进行坐标转换时, 需要选定一个参考系, 本文叙述了坐标转换在高速公路非正常停车系统 中的具体应用，即通过雷达传送回来的数据进行位置坐标的转换。

在高速公路上, 雷达对公路上行驶的车辆进行定位, 在对雷达信号进行处理的时候, 经常遇 到坐标转换问题, 坐标数据转换后, 才方便把数据送给下一级进行处理, 本系统就采用基于 CORDIC 的坐标转换思想, 对该系统雷达得到的坐标数据进行转换, 基于一种流水线思想, 并 在 FPGA 上得到了验证。 


\section{CORDIC 算法原理}

CORDIC 算法是 Vloder 与 1959 年设计航空导航控制系统过程中更提出的, 为了解决导航系统 中的三角函数表、反三角函数和开方等比较复杂的计算问题。1971, Walther 提出了一种统 一的 CORDIC 算法形式。

传统的坐标转换算法在精度, 效率等方面性能不能同时兼顾, 基于 CORDIC 算法的坐标转换是 一种数学计算的逼近方式, 最终可以分解为一系列的移位操作和加减法运算, 基于 FPGA 硬件 平台来对该算法很容易实现, 并且利用一种流水线结构来提高运算的速度, 应用到该系统中, 可以充分提高效率和实时性。

从直角坐标到极坐标的转换, cordic 的想法很直接, 将一个点与坐标原点连线的直线进行旋 转, 旋转后的该点纵坐标如果变为 $0^{\circ}$, 那说明旋转的角度就是为极角, 一个坐标点的在平 面直角坐标系下为 ( $\mathrm{x}, \mathrm{y}$ ), 则基于坐标转换公式: $\left(\begin{array}{c}X q \\ Y q\end{array}\right)=\left(\begin{array}{cc}\cos \theta & -\sin \theta \\ \sin \theta & \cos \theta\end{array}\right)\left(\begin{array}{l}x \\ y\end{array}\right)$, 该式子进行 化简可以得到 $\left(\begin{array}{l}X q \\ Y q\end{array}\right)=\cos \theta\left(\begin{array}{cc}1 & -\tan \theta \\ \tan \theta & 1\end{array}\right)\left(\begin{array}{l}x \\ y\end{array}\right)$, 因为 $\cos \theta$ 只是改变该点向量的模长并不改变 角度, 所以已进一步可以得到 $\left\{\begin{array}{l}X p=x-y \tan \theta \\ Y \mathrm{p}=x \tan \theta+\mathrm{y}\end{array}\right.$, 最后此时得到的为 cordic 的一般公式: $\left\{\begin{array}{l}x_{i+1}=x_{i}-d_{i} y_{i} 2^{-i} \\ y_{i+1}=y_{i}+d_{i} x_{i} 2^{-i} \\ z_{i+1}=z_{i}-d_{i} \tan ^{-1} 2^{-i}\end{array}\right.$

(其中当 $y_{i} \leq 0$ 时 $d_{i}=1$, 当 $y_{i}>0$ 时 $d_{i}=-1$ ), 经过 $\mathrm{n}$ 次旋转, 最终可以得到式子 $\left\{\begin{array}{l}X n=A n \sqrt{x_{\mathrm{o}}{ }^{2}+y_{\mathrm{o}}{ }^{2}} \\ Y n=0 \\ Z n=z_{\mathrm{O}}+\tan ^{-1}\left(\frac{y_{\mathrm{O}}}{x_{\mathrm{o}}}\right)\end{array}\right.$.

其中 $\mathrm{An}=\sqrt{\left(1+2^{-2 * 1}\right)} * \sqrt{\left(1+2^{-2 * 2}\right)} * \sqrt{\left(1+2^{-2 * 3}\right)} \cdots \cdots \cdot \sqrt{\left(1+2^{-2 * i}\right)}$ (其中 $\left.\mathrm{i}=1,2,3 \cdots \mathrm{n}-1\right)$ 。 该种算法称为伪旋转, 省去的部分使得模长发生变化, 所以需要对模长进行补偿。 对 (1) 式子前期进行数据检验, 检验数据如下表

\begin{tabular}{ccccc}
\hline $\mathrm{i}$ & $\mathrm{Xi}$ & $\mathrm{Yi}$ & $2^{-\mathrm{i}}$ & $\mathrm{Zi}$ \\
\hline 0 & 1 & 2 & 1 & 0 \\
1 & 3 & 1 & 0.5 & 45 \\
2 & 3.5020 & -0.5011 & 0.25 & 70.1458 \\
3 & 3.6618 & 0.3685 & 0.125 & 56.5174 \\
\hline
\end{tabular}

数据检验误差在 $5 \%$ 以内, $x_{n}$ 最终输出为极径, 相对初始模长扩大了 An 倍, 对 $\mathrm{Zn}$ 进行一定处 理方可变为极角。 


\section{CORDIC 应用}

本系统框图如下:

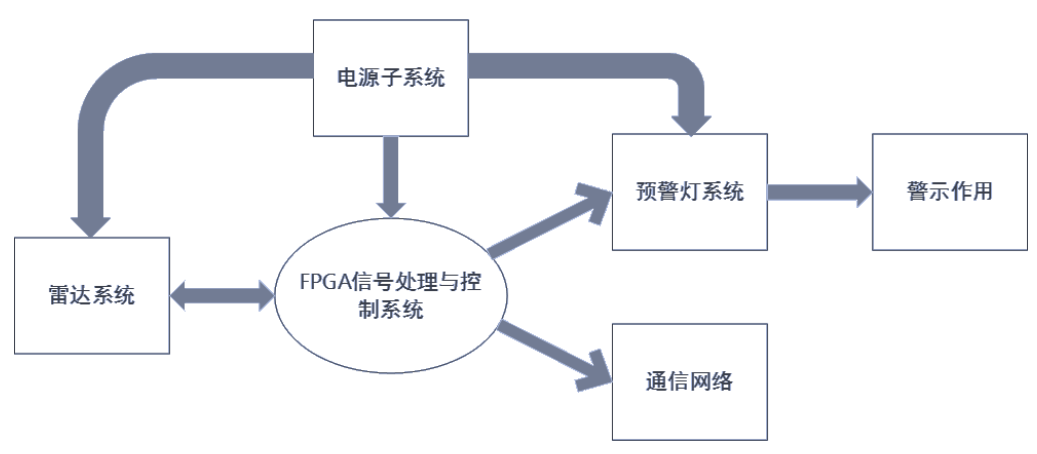

图 1 系统框图

该系统主控系统接收到高速路段雷达系统检测到的停止车辆的坐标信息, 主控系统经过处理 将命令通过光纤通信传到各个无线通信模块, 并通过无线通信将命令传达到各个外围电路, 即各个高亮指示灯, 在该系统中对坐标的处理转换在 fpga 实现, 对坐标转换来说, 需要进一 步改写算法式子, 易于 FPGA 硬件的实现, 改写为统一的算法形式

$\left\{\begin{array}{l}x_{i+1}=x_{i}-\mu \operatorname{di}\left(2^{-i} y_{i}\right) \\ y_{i+1}=y_{i}+\operatorname{di}\left(2^{-i} x_{i}\right) \\ z_{i+1}=z_{i}+d_{i} \theta_{i}\end{array}\right.$

(3) ( $\mu$ 在不同的系统中值不同)

对统一的算法式子有三个加法, 所以需要三个加法器, 并且加上 $i$ 的值, 需要进行移位操作, 每一次迭代, 都会有个相对固定的角度的累加, 这个角度分别是 2 的-i 次方对应的角度值, 用查找表实现, 所以需要数据的储存单元 LUT, cordic 的突出优点就在于此, 不需要复杂的 单元。

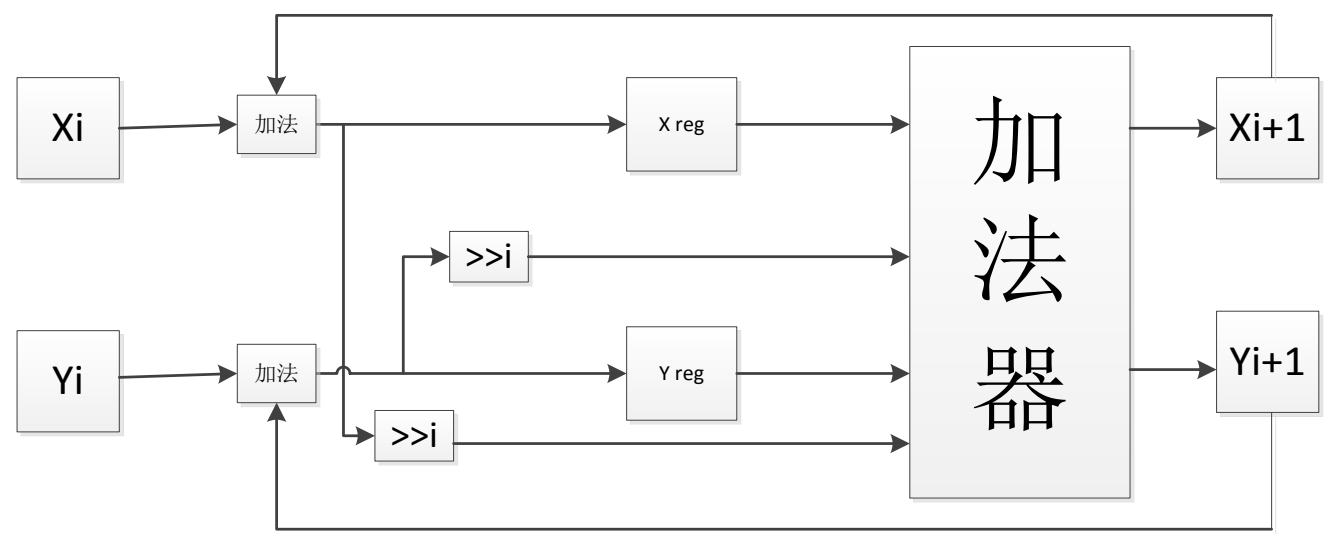

图 2 硬件处理单元

本系统应用在高速公路中, 要求速度比较快, 所以采用一种流水线结构的 cordic 思想, 流水 线技术就是通过在长延时的组合逻辑电路中插入触发器产生的。其优势: 减小毛刺信号的产 生; 提高系统的时钟速率 
流水线的核心思想: 将较大的组合逻辑分解为较小的 $N$ 块, 通过适当的方法平均分配组合逻 辑, 然后在中间插入触发器, 并和原触发器使用相同的时钟, 就可以避免在两个触发器之间 出现过大的延时, 消除速度瓶颈, 以提高电路的工作频率。即, 原设计速度受限部分用一个 低速时钟周期实现, 采用流水线技术插入触发器后, 可用 $\mathrm{N}$ 个高速时钟周期实现。增加流水 寄存器, 增大面积来提高速度, 有效的提高了处理速度, 使得 $\mathrm{N}$ 个单元处理变为了一个单元 处理。结构如下:

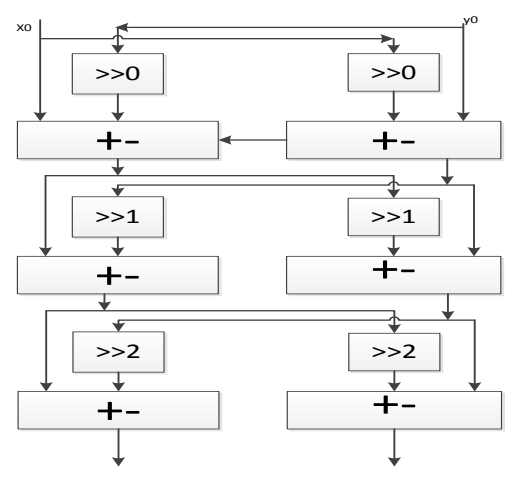

图 3 流水线结构

通过在 ISE 软件中，对加法器使用流水线技术，生成 RTL 图，所得 RTL 图如下：

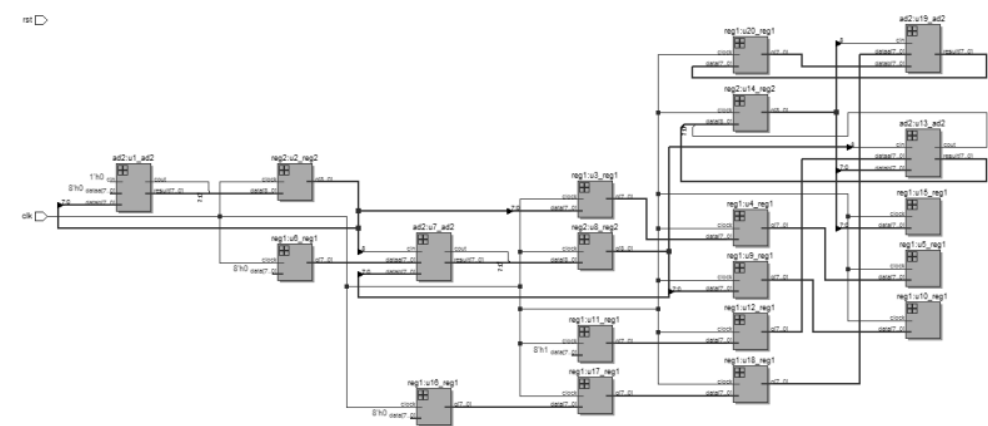

图 432 位加法器流水线结构

利用 xilinx 平台的 spartan6 测试该算法，并通过 modelsim 进行仿真，仿真结果如下：

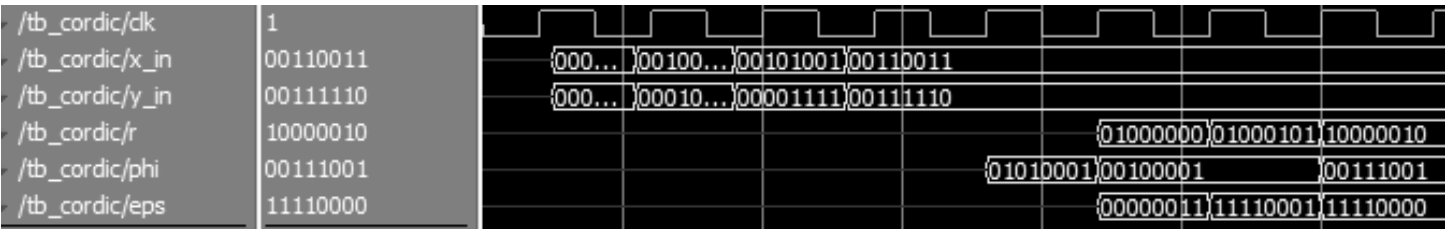

图 5 Modesim 仿真

\section{4. 结语}

利用流水线结构的 CORDIC 算法思想, 对本系统进行坐标转换操作, 误差比较合理, 并且能够 达到速度要求。 


\section{参考文献}

[1] 李滔, 韩月秋.基于 CORDIC 算法的雷达信号坐标变换处理器.现代雷达, 1999.4

[2] U. Meyer-Baese.刘凌译 数字信号处理的 FPGA 实现.清华大学出版社.2011

[3] 谈宜育, 市文兵, 李元.一种基于 COREDI 算都发的坐标变换电路.数据采集与处理.2001

[4] XU Guo ku, H Chen. FPGA Realization of Coordinate Conversion Solution Based on CORDIC Algorithm. Modern Electronics Technique.2006

[5] 李庆峰.CORDIC 算法硬件电路实现及改进.电子与封装.2014(1):31-33

[6] 郑杰然. CORDIC 算法基本原理研究.电子世界.2014（8）：199-199

[7] K Seki.CORDIC OPERATIONAL CIRCUIT AND METHOD.EP.2014

[8] YL Chen.FPGA Realization of Transformation Circuit between Rectangular Coordinate and Spherical Coordinate Based on CORDIC. Shipboard Electronic Countermerasure.2011

[9] Z Juntao,M Wenbo. Implementation of General CORDIC IP Core Based on FPGA. Microcomputer Information.2008,3:606-608

[10]M Chinnathambi, N Bharanidharan, S Rajaram. FPGA implementation of fast and area efficient CORDIC algorithm. International Conference on Communication \&Network Technologies.2014

\section{References}

[1] Li Tao, Han Yueqiu. Radar signal coordinate transformation based on CORDIC processor. Modern radar.1999.4

[2] U. Meyer-Baese. Liu Ling Translation. Digital signal processing on FPGA. Tsinghua University Press.2011

[3] Tan Yi yu, Bian Xiawen, Li Yuan. Based on coordinate conversion circuit COREDI Operators are issued. Data Acquisition and Processing.2001

[4] XU Guo ku, H Chen. FPGA Realization of Coordinate Conversion Solution Based on CORDIC Algorithm. Modern Electronics Technique.2006

[5] Li Xiaofeng. CORDIC algorithm hardware circuit and Improvement. Electronics and Packaging. 2014(1):31-33

[6] Zheng Jieran. CORDIC algorithm for the basic principles of research. Electronic world. .2014 (8) : 199-199

[7] K Seki. CORDIC OPERATIONAL CIRCUIT AND METHOD.EP.2014

[8] YL Chen. FPGA Realization of Transformation Circuit between Rectangular Coordinate and Spherical Coordinate Based on CORDIC. Shipboard Electronic Countermerasure.2011

[9] Z Juntao, M Wenbo. Implementation of General CORDIC IP Core Based on FPGA. M Chinnathambi, N Bharanidharan, S Rajaram. FPGA implementation of fast and area efficient CORDIC algorithm. International Conference on Communication \&Network Technologies.2014 